\section{ORIGINAL RESEARCH}

\author{
D. Dai \\ Y.H. Ding \\ R. Kadirvel \\ D.A. Lewis \\ D.F. Kallmes
}

\title{
Experience with Microaneurysm Formation at the Basilar Terminus in the Rabbit Elastase Aneurysm Model
}

\begin{abstract}
BACKGROUND AND PURPOSE: Intracranial aneurysms have been induced in mice, rats, rabbits, and primates through carotid artery ligation. We reviewed our experience with RCCA ligation to quantify the rate of aneurysm formation in rabbits.
\end{abstract}

\begin{abstract}
MATERIALS AND METHODS: In 30 consecutive New Zealand white rabbits, the RCCA was ligated during surgery to create elastase-induced aneurysms. The basilar artery and its bifurcation were harvested at various time points after surgery, including 8 weeks $(n=5), 13$ weeks $(n=3), 14$ weeks $(n=2)$, 16 weeks $(n=4), 17$ weeks $(n=4), 30$ weeks $(n=6)$, and 33 weeks $(n=6)$. All specimens were embedded in paraffin and sectioned at $5 \mu \mathrm{m}$ in a coronal orientation, to show the basilar bifurcation and its branches. All sections were stained with $\mathrm{HE}$. After the sections were evaluated and the photomicrographs were taken, the sections were de-stained and re-stained with WVG staining for elastin.
\end{abstract}

RESULTS: The IEL was intact and continuous at the BT in all 30 rabbits, as was the medial layer. No bulge-like localized dilation, to suggest microaneurysm or nascent aneurysm formation, was observed at the BT in any subject. There were small $(0.08 \pm 0.02 \mathrm{~mm}$ in diameter) concave structures along the $\mathrm{P} 1$ segments in $5(16.7 \%)$ of 30 rabbits. On adjacent tissue sections, each of these 5 structures was shown to be branch vessels with intact IELs.

CoNCLUSIONS: In our rabbit model, unilateral RCCA ligation does not induce microaneurysm formation.

\begin{abstract}
ABBREVIATIONS: $\mathrm{BT}=$ basilar terminus; $\mathrm{CCA}=$ common carotid artery; $\mathrm{DSA}=$ digital subtraction angiography; $\mathrm{ECA}=$ external carotid artery; $\mathrm{HE}=$ hematoxylin-eosin; ICA = internal carotid artery; $\mathrm{IEL}=$ internal elastic lamina; PcomA = posterior communicating artery; RCCA = right common carotid artery; $\mathrm{VVG}=$ Verhoeff-van Gieson
\end{abstract}

I ntracranial aneurysms have been induced in rats, mice, and primates through carotid artery ligation. ${ }^{1-5}$ These intracranial aneurysms form in areas of elevated blood flow, which results from the carotid artery ligation with subsequent recruitment of flow across communicating arteries. Recently, Gao et $\mathrm{al}^{6} \mathrm{demon}^{-}$ strated consistently elevated basilar artery flow rates following either unilateral or bilateral carotid artery ligation. In that same study, the authors noted nascent aneurysm formation at the BT, apparently as a result of the elevated hemodynamic stress in the basilar apex from carotid artery ligation.

We routinely ligate the RCCA during surgery to create dilated arterial segments in the proximal RCCA. We have noted on angiography that after RCCA ligation, the basilar artery via the right PcomA not only feeds the ipsilateral anterior circulation of the brain but also supplies blood flow retrograde down the ipsilateral ICA to the ECA branches. The ECA territory in the rabbit supplies a substantial amount of soft tissue,

Received April 13, 2009; accepted after revision July 8.

From the Neuroradiology Research Laboratory, Department of Radiology, Mayo Clinic, Rochester, Minnesota.

This project was partially supported by National Institutes of Health grant NS42646.

Paper previously presented at: Annual Meeting of the American Society of Neuroradiology, May 16-21, 2009, Vancouver, British Columbia, Canada.

Please address correspondence to David F. Kallmes, MD, Mayo Clinic, 200 First St SW, Rochester, MN 55905; e-mail: kallmes.david@mayo.edu

Indicates open access to non-subscribers at www.ajnr.org

DOI 10.3174/ajnr.A1823 with markedly greater demand for blood than the small intracranial circulation. These hemodynamic factors result in markedly increased flow across the right PcomA, from the basilar artery to the ICA and ECA, which might predispose the experimental subjects to aneurysm formation.

On the basis of the recent report of microaneurysm formation after RCCA ligation in rabbits by Gao et al, ${ }^{6}$ we prospectively performed histologic evaluation of the BT in subjects that had undergone RCCA ligation as part of elastase aneurysm surgery, to confirm the ability of RCCA ligation to induce microaneurysms.

\section{Materials and Methods}

Elastase-induced aneurysms were created by using vessel ligation and elastase incubation in the RCCA in 30 consecutive New Zealand white rabbits. All procedures were approved by the Institutional Animal Care and Use Committee at our institution. Detailed procedures for aneurysm creation have been described elsewhere. ${ }^{7,8}$ Briefly, New Zealand white rabbits (3-4 kg) were anesthetized with an intramuscular injection of ketamine, xylazine, and acepromazine $(75,5$, and $1 \mathrm{mg} / \mathrm{kg}$, respectively). With a sterile technique, the RCCA was exposed and ligated distally. A 5F sheath was advanced retrograde in the RCCA to a point approximately $3 \mathrm{~cm}$ cephalad to the origin of the RCCA. A 3F Fogarty balloon (Baxter Healthcare, Irvine, California) was advanced through the indwelling sheath to the origin of the RCCA at its junction with the right subclavian artery. The balloon was inflated with just enough iodinated contrast material to achieve flow arrest in the RCCA. Porcine elastase mixed with iodinated contrast material was incubated in the dead space of the RCCA, above the 

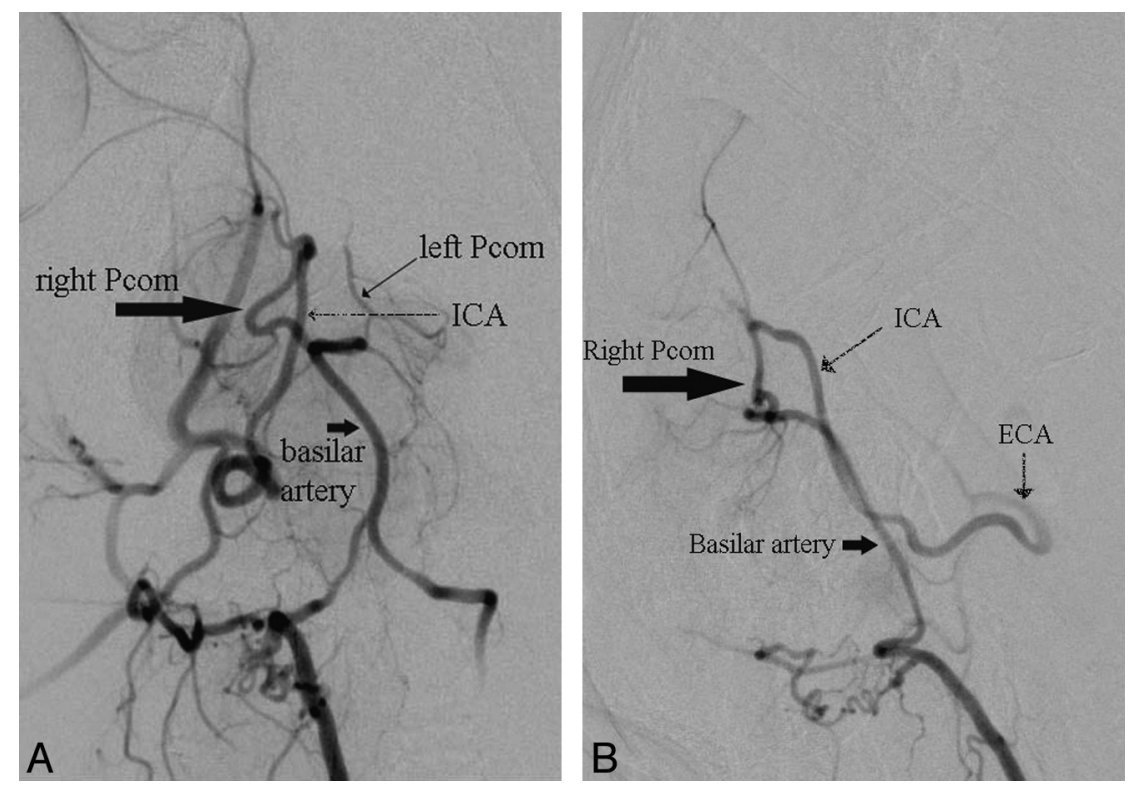

Fig 1. A, DSA of the right vertebral artery shows the basilar artery (short arrow) and bilateral PcomAs (long arrows). The right PcomA is tortuous and dilated and nearly as large as the basilar artery, compared with the left PcomA (thin long arrow). B, Lateral DSA from the same rabbit subject shows marked enlargement of the right PcomA, which fills, in retrograde fashion, the ECA branches

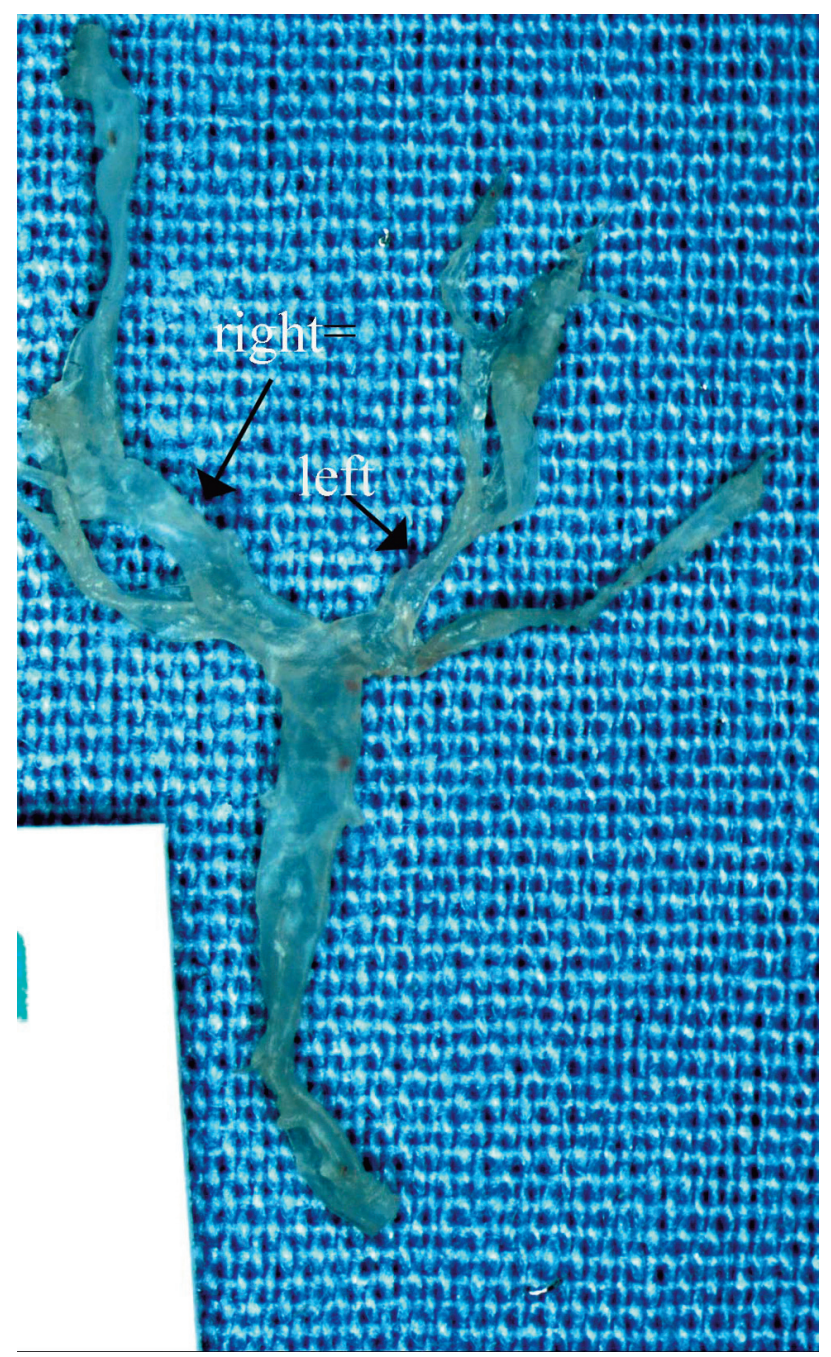

Fig 2. A photograph of the basilar artery and its branches shows that both PcomAs are present and that the right PcomA (long arrow) is larger than the left one (short arrow). inflated balloon, through a microcatheter. After incubation with the elastase solution, the balloon and sheath were removed, and the RCCA was ligated below the sheath entry site.

The animals used in this study were subjects used for testing aneurysm devices. We did not alter subject numbers and the duration of the ligation for the basilar artery studies, but we believe that these samples, given their large number and long duration, are adequate to answer our research question.

\section{Tissue Harvest}

In selected subjects, at the time of tissue harvest, injection of the right vertebral artery was performed to assess the degree of hypertrophy of the PcomA and associated arteries (Fig 1). After deep sedation, the rabbits were euthanized. Tissues were harvested at various time points following vessel ligation, including 8 weeks $(n=5), 13$ weeks $(n=3)$, 14 weeks $(n=2), 16$ weeks $(n=4), 17$ weeks $(n=4), 30$ weeks ( $n=$ $6)$, and 33 weeks $(n=6)$. The basilar artery and its branches were carefully dissected free from surrounding tissues and removed. All specimens were fixed in $10 \%$ neutral buffered formalin for at least 24 hours. Gross inspection of the arterial tree was performed to determine the presence of PcomAs in all cases. The arteries were then embedded in paraffin for histologic examination.

\section{Histologic Processing}

The fixed vessels were dehydrated in an ascending series of ethanol cleared in xylene and embedded in paraffin. Vessels were then sectioned at $5 \mu \mathrm{m}$ in a coronal orientation, to show the basilar bifurcation and its branches. The first section was collected once the investigator observed the bifurcation of basilar artery; then all of the serial sections were collected until the bifurcation disappeared.

\section{Staining}

HE. All sections of each sample were stained with HE for conventional histopathologic evaluation. After the HE staining, sections were evaluated, the photomicrographs were taken, and the slides were 


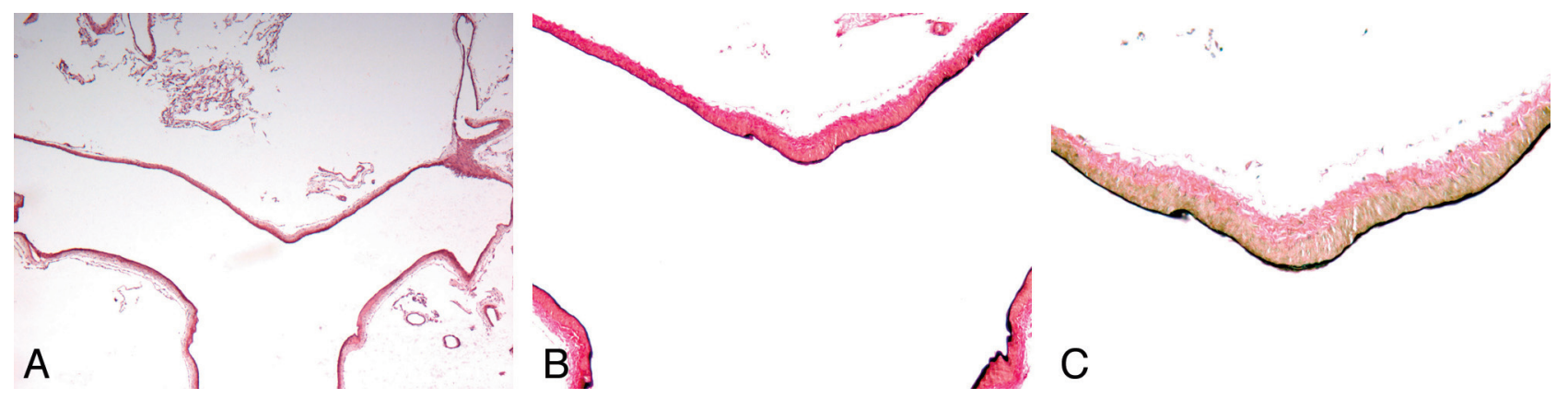

Fig 3. Typical photomicrographs of the $B T$ show that the IEL is intact and continuous at the BT. $A$, The basilar artery and its bifurcation (HE, original magnification $\times 40)$. $B$ and $C$, The IEL (black line) at the BT is intact and continuous $(B$, VG , original magnification $\times 100)(C$, VVG, original magnification $\times 200)$. The medial layer, immediately underneath the IEL and stained light brown, is also intact and continuous. No bulge-like localized dilation or nascent aneurysm formation is observed.
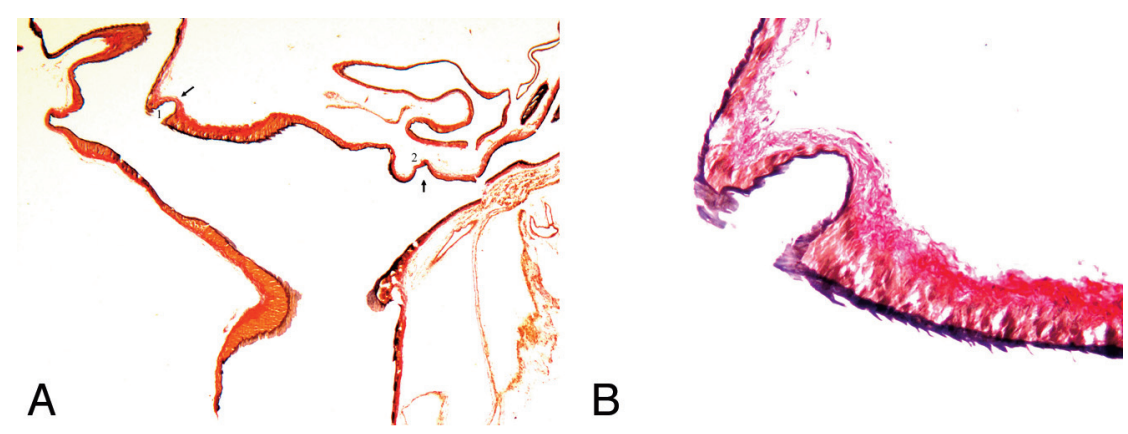

B
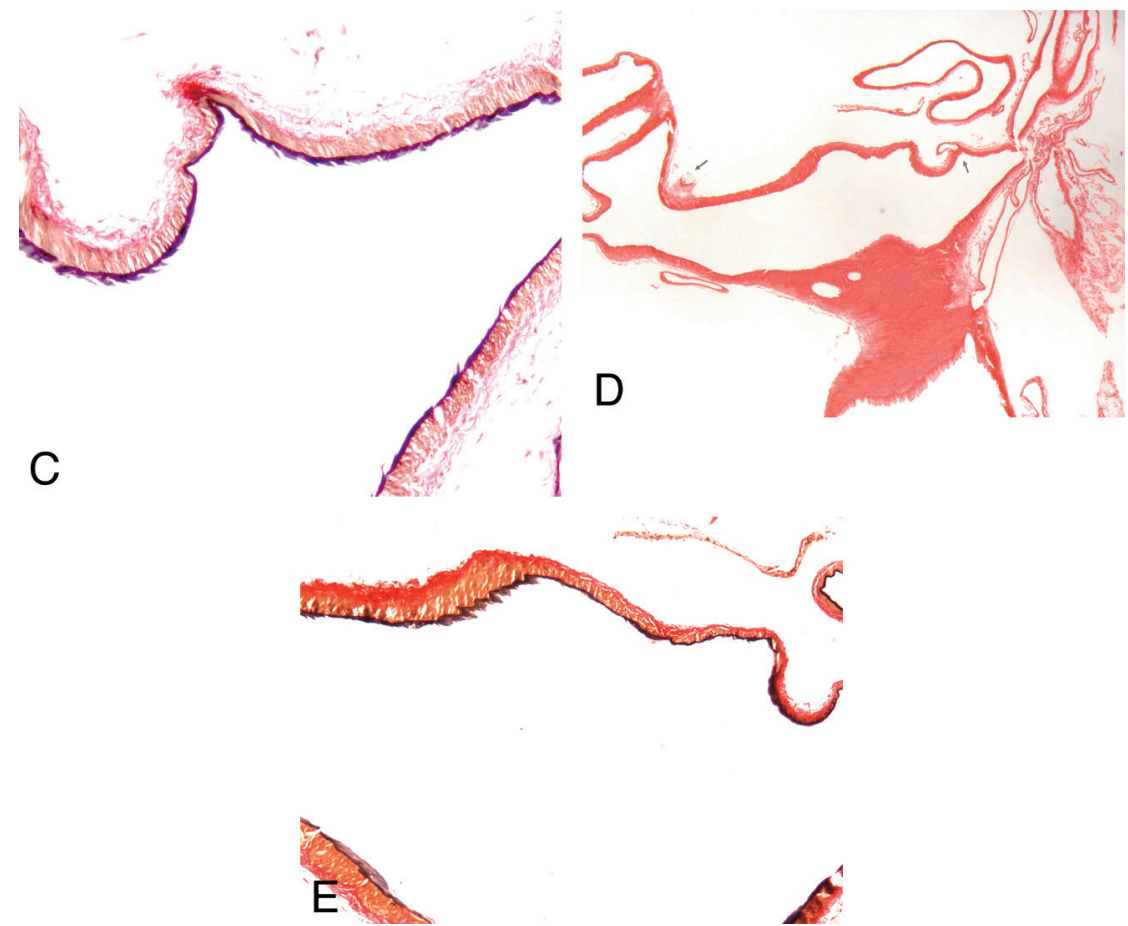

Fig 4. Photomicrographs of the BT from a 30-week subject. $A$, The basilar artery, BT, and 2 concave structures along the $\mathrm{P} 1$ segments labeled as 1 and 2 (arrows) for correlation with higher magnification views in $B$ and $C(\mathrm{VVG}$, original magnification $\times 40)$. $B$, The concave structure 1 from $A$, with an intact IEL, is the black line along the luminal side of the wall, contiguous with the adjacent vessel wall and with the intact but thinned media (VVG, original magnification $\times 200)$. $C$, Concave structure 2 from $A$, with an intact IEL (VVG, original magnification $\times 200)$. $D$, The serial (adjacent) section of $A$ shows that the concave structures in $A-C$ are branch vessels (arrows) (HE, original magnification $\times 40)$. $E$, Intact and continued IEL of the BT (VVG, original magnification $\times 200)$.

placed in $70 \%$ alcohol with $1 \%$ hydrochloric acid to remove the HE stain. The slides were then washed in tap water for 10 minutes and stained with VVG.

VVG. The de-stained HE sections were placed in VVG staining solutions for 15 minutes. They were rinsed in running distilled water for 10 minutes, after which they were differentiated in $2 \%$ ferric chloride with a microscope. After being rinsed in running distilled water, the slides were rinsed in $95 \%$ alcohol for 30 seconds and counterstained in filtered van Gieson solution for 1 minute 30 seconds. Finally, the slides were dehydrated in methanol and acetone, cleared in xylene, and mounted with Shandon EZ-Mount medium (Thermo Scientific, Waltham, Massachusetts). 


\section{Results}

Typical angiographic findings after RCCA ligation are shown in Fig 1. Brisk flow is seen when injecting the vertebral artery across the tortuous and dilated right PcomA to fill the right cerebral hemisphere, which represents only a small amount of flow compared with the large volume that travels retrograde in the ICA to fill the ECA branches (Fig 1). Gross examination showed PcomAs to be present and asymmetrically enlarged on the right side in all cases (Fig 2).

\section{Histologic Evaluation of the BT}

The IEL was intact and continuous at the BT in all (30/30) rabbits, as was the medial layer in all cases (Fig $3 A-C$ ). No bulge-like localized dilation associated with a missing IEL, to suggest microaneurysm or nascent aneurysm formation, was observed at the BT in any subject. There were small (0.08 \pm $0.02 \mathrm{~mm}$ in diameter) concave structures along the P1 segments or at the BT area in $5(16.7 \%)$ of 30 rabbits (Fig $4 A$ ). These concave structures had intact IELs, which were contiguous with the adjacent vessel wall as well as the intact but thinned media (Fig 4B). Each of these 5 concave structures was shown to be a branch vessel (Fig $4 C,-D$ ) on adjacent tissue sections.

\section{Discussion}

Increased flow and hemodynamic stress have been shown to be important factors in the formation of aneurysms in various animal models. ${ }^{2,5,6,9,10}$ Previous reports indicated that increased hemodynamic stress reliably resulted in microaneurysm formation in small animals. ${ }^{2,11}$ Recently, Gao et $\mathrm{al}^{6}$ reported that uni- or bilateral CCA ligation induced microaneurysm formation at the BT in rabbits. A relationship between aneurysm formation and elevated basilar artery flow was noted in that study, with higher indices of aneurysm formation seen with bilateral compared with unilateral CCA occlusion.

A review of consecutive animals subjected to chronic unilateral CCA occlusion in our own laboratory failed to uncover any evidence of microaneurysm formation along the BT. We did detect small concave structures along the P1 segments or at the BT histologically in a minority of cases, but each of these was shown to be a branch vessel rather than a nascent aneurysm. Evidence that these structures represented branch vessels included intact IELs and direct contiguity to branches identified on adjacent histologic sections. Thus, at least in the New Zealand white rabbits used in our laboratory, elevated flow does not appear to induce nascent aneurysm formation.

Multiple prior reports are available regarding hemodynamic alterations leading to aneurysm formation in animal models. Hashimoto et $\mathrm{al}^{1}$ reported that the occurrence of aneurysms in the circle of Willis was induced by unilateral CCA ligation and that hypertension was low, though hypertension could increase the incidence of aneurysm formation. Gao et al, ${ }^{6}$ however, demonstrated that the occurrence of an intracranial aneurysm was $100 \%$ by using unilateral or bilateral ligation of the CCA, even without hypertension induction. Although hemodynamics plays a big role in aneurysm formation, the precise mechanism still remains to be determined.

There are several potential reasons to explain the disparate results between our series and that of Gao et al. ${ }^{6}$ Different lineages of the New Zealand white rabbit may have differential susceptibilities to aneurysm formation. Further, in some cases, Gao et $\mathrm{al}^{6}$ performed bilateral CCA ligation, whereas we performed only unilateral ligation. Gao et $\mathrm{al},{ }^{6}$ however, did report microaneurysm formation even with unilateral ligation. We did not perform a "nascent aneurysm index," as reported by Gao et al, ${ }^{6}$ because we saw no evidence of IEL loss in any of our subjects. Finally, it remains possible that at least in some of their cases, branch arteries artifactually simulated microaneurysms.

There are several limitations in the current study: First, the comparison of aneurysm formation induced through bilateral ligation of the CCA and/or hypertension was not performed. Second, we did not measure the hemodynamic change at the BT after unilateral RCCA ligation. Third, we did not perform a sham control. We acknowledge that sham controls might have added information regarding the branch vessels that we identified - that is, whether these branches were normal-sized or hypertrophied. However, because our research focus was the development of aneurysms after carotid ligation and because we identified no aneurysms in the ligated group, we contend that the addition of a sham control group would add little value. Last, we used rabbit subjects that had undergone carotid ligation as part of elastase-induced aneurysm research protocols, rather than subjects specifically dedicated to intracranial aneurysm research. As such, our time points were prespecified on the basis of the elastase aneurysm projects and the resultant number of subjects varied slightly across time points.

\section{Conclusions}

In this study, unilateral RCCA ligation did not induce microaneurysm formation at the BT in rabbits.

\section{References}

1. Hashimoto N, Handa H, Hazama F. Experimentally induced cerebral aneurysms in rats. Surg Neurol 1978;10:3-8

2. Hashimoto N, Handa H, Nagata I, et al. Experimentally induced cerebral aneurysms in rats. Part V. Relation of hemodynamics in the circle of Willis to formation of aneurysms. Surg Neurol 1980;13:41-45

3. Hashimoto N, Handa H, Nagata I, et al. Animal model of cerebral aneurysms: pathology and pathogenesis of induced cerebral aneurysms in rats. Neurol Res 1984;6:33-40

4. Hashimoto N, Kim C, Kikuchi H, et al. Experimental induction of cerebral aneurysms in monkeys. J Neurosurg 1987;67:903-05

5. Morimoto M, Miyamoto S, Mizoguchi A, et al. Mouse model of cerebral aneurysm: experimental induction by renal hypertension and local hemodynamic changes. Stroke 2002;33:1911-15

6. Gao L, Hoi Y, Swartz DD, et al. Nascent aneurysm formation at the basilar terminus induced by hemodynamics. Stroke 2008;39:2085-90

7. Altes TA, Cloft HJ, Short JG, et al. 1999 ARRS Executive Council Award: creation of saccular aneurysms in the rabbit-a model suitable for testing endovascular devices. American Roentgen Ray Society. AJR Am J Roentgenol 2000;174:349-54

8. Ding YH, Danielson MA, Kadirvel R, et al. Modified technique to create morphologically reproducible elastase-induced aneurysms in rabbits. Neuroradiology 2006;48:528-32

9. Kadirvel R, Ding YH, Dai D, et al. The influence of hemodynamic forces on biomarkers in the walls of elastase-induced aneurysms in rabbits. Neuroradiology 2007;49:1041-53

10. Meng H, Swartz DD, Wang Z, et al. A model system for mapping vascular responses to complex hemodynamics at arterial bifurcations in vivo. Neurosurgery 2006;59:1094-100, discussion 1100-01

11. Coutard M. Experimental cerebral aneurysms in the female heterozygous blotchy mouse. Int J Exp Pathol 1999;80:357-67 